\title{
Algebraic cubature by linear blending of elliptical arcs*
}

\author{
Gaspare Da Fies, Alvise Sommariva ${ }^{1}$ and Marco Vianello ${ }^{1}$
}

April 28, 2013

\begin{abstract}
We construct a cubature formula of algebraic degree of exactness $n$ with $n^{2} / 2+\mathcal{O}(n)$ nodes, on the bidimensional domains generated by linear blending of two arcs of ellipses corresponding to the same angular interval. The construction is based on recent results on "subperiodic" trigonometric quadrature. Our formula generalizes several recent cubature formulas on standard circular sections. Among its numerous possible applications, we quote for example integration of functions with singularities, and integration on nonstandard circular sections arising in optical design or in meshfree methods with compactly supported radial bases.
\end{abstract}

2000 AMS subject classification: $65 \mathrm{D} 32$.

Keywords: algebraic cubature, subperiodic trigonometric quadrature, product Gaussian quadrature, linear blending of elliptical arcs, singular integrand, nonstandard circular/elliptical sections, obscured and vignetted pupils, meshfree methods.

\section{Introduction}

In some recent papers, "subperiodic" trigonometric interpolation and quadrature have been studied, i.e., interpolation and quadrature formulas exact for

$$
\mathbb{T}_{n}([-\omega, \omega])=\operatorname{span}\{1, \cos (k \theta), \sin (k \theta), 1 \leq k \leq n, \quad \theta \in[-\omega, \omega]\},
$$

where $0<\omega \leq \pi$; cf. $[2,4,5,6]$. In particular, a trigonometric Gaussian quadrature formula on subintervals of the period has been provided. It is related by the simple nonlinear transformation

$$
\theta=2 \arcsin (x \sin (\omega / 2)),
$$

*Supported by the "ex-60\%" funds of the University of Padova, and by the GNCSINdAM.

${ }^{1}$ Dept. of Mathematics, University of Padova, Italy

e-mail: alvise,marcov@math.unipd.it 
to an algebraic Gaussian formula on $(-1,1)$, and can be effectively implemented in Matlab by Gautschi's OPQ (Orthogonal Polynomials and Quadrature) suite [13]. For the reader's convenience, we report the main result of [5] for an arbitrary subinterval of the period:

Proposition 1 Let $\left\{\left(\xi_{j}, \lambda_{j}\right)\right\}_{1 \leq j \leq n+1}$, be the nodes and positive weights of the algebraic Gaussian quadrature formula for the weight function

$$
w(x)=\frac{2 \sin (\omega / 2)}{\sqrt{1-x^{2} \sin ^{2}(\omega / 2)}}, x \in(-1,1), \omega \in(0, \pi] .
$$

Then for $0<\beta-\alpha \leq 2 \pi$ the following trigonometric Gaussian quadrature formula holds

$$
\int_{\alpha}^{\beta} f(\theta) d \theta=\sum_{j=1}^{n+1} \lambda_{j} f\left(\theta_{j}+\mu\right), \quad \forall f \in \mathbb{T}_{n}([\alpha, \beta]), \quad \mu=\frac{\alpha+\beta}{2},
$$

where

$$
\theta_{j}=2 \arcsin \left(\xi_{j} \sin (\omega / 2)\right) \in(-\omega, \omega), \quad j=1,2, \ldots, n+1, \omega=\frac{\beta-\alpha}{2} .
$$

Formula (4) opens the way to construct algebraic cubature formulas of product type on several arc-related sections of planar, surface and solid regions. The first steps made in $[4,5]$ have led to product Gaussian formulas on planar circular segments, (annular) sectors, zones, lenses and bubbles.

A key aspect is efficient implementation of (4), since unlike algebraic Gaussian formulas the nodes and weights cannot be tabulated in a reference subinterval, and have to be recomputed when $\omega$ changes. In $[5,6]$ a Chebyshev moment-based algorithm has been used; an alternative method could resort to the more general sub-range Jacobi polynomials approach, studied in [14].

In the present paper we provide product type algebraic cubature formulas for the planar regions generated by convex combination of the polar parametrizations (with respect to the centers) of two arcs of ellipses corresponding to equal length angular intervals. This procedure is also known in the CAGD and transfinite interpolation literature as (linear) blending of the two arcs; see, e.g., [9]. A degenerate relevant case is generalized elliptical (circular) sectors, where the vertex does not coincide, in general, with the center of the ellipse. We stress that the present formulas generalize some previous work concerning cubature on standard circular sections, since it contains as special cases: circular (annular) sectors, circular zones and segments, symmetric lenses.

Such blending cubature formulas have several possible applications. We discuss three of them: integration on sections of a lens by a line or an arc, 
which can be relevant for example to meshfree methods with compactly supported radial bases; integration on obscured and vignetted pupils, which is relevant to optical design; a general purpose treatment of singularities by clustering of cubature points.

\section{Linear blending of elliptical arcs}

In this section we construct an algebraic cubature formula of product type on planar domains generated by linear blending of two elliptical arcs, which have as a special case generalized elliptical sectors. Let

$$
P(\theta)=A_{1} \cos (\theta)+B_{1} \sin (\theta)+C_{1}, \quad Q(\theta)=A_{2} \cos (\theta)+B_{2} \sin (\theta)+C_{2},
$$

$\theta \in[\alpha, \beta]$, be two trigonometric planar curves of degree one,

$$
A_{i}=\left(a_{i 1}, a_{i 2}\right), \quad B_{i}=\left(b_{i 1}, b_{i 2}\right), \quad C_{i}=\left(c_{i 1}, c_{i 2}\right), \quad i=1,2,
$$

being suitable bidimensional vectors (with $A_{i}, B_{i}$ not all zero), with the important property that the curves are both parametrized on the same angular interval $[\alpha, \beta], 0<\beta-\alpha \leq 2 \pi$. It is not difficult to show, by a possible riparametrization with a suitable angle shift when $A_{i}$ and $B_{i}$ are not orthogonal, that these curves are arcs of two ellipses centered at $C_{1}$ and $C_{2}$, respectively.

Consider the domain

$$
\Omega=\{(x, y)=U(t, \theta)=t P(\theta)+(1-t) Q(\theta), \quad(t, \theta) \in[0,1] \times[\alpha, \beta]\},
$$

which is the transformation of the rectangle $[0,1] \times[\alpha, \beta]$ obtained by convex combination (linear blending) of the $\operatorname{arcs} P(\theta)$ and $Q(\theta)$. The map $U$ is analytic in $(t, \theta)$. A special degenerate case is when for example $A_{1}=B_{1}=$ $(0,0)$, so that $C_{1}$ becomes the vertex of a "generalized elliptical sector" (not coinciding, in general, with the other "center" $C_{2}$ ); see Figure 2.

Assume that the linear blending transformation $U$ is injective in the interior of the rectangle: then, by topological degree theory its Jacobian determinant doesn't change sign; cf., e.g., [18, Ch. III]. Writing $P(\theta)=$ $\left(p_{1}(\theta), p_{2}(\theta)\right)$ and $Q(\theta)=\left(q_{1}(\theta), q_{2}(\theta)\right)$ in (5), we get

$$
\begin{gathered}
J U(t, \theta)=\left(\begin{array}{cc}
p_{1}(\theta)-q_{1}(\theta) & t p_{1}^{\prime}(\theta)+(1-t) q_{1}^{\prime}(\theta) \\
p_{2}(\theta)-q_{2}(\theta) & t p_{2}^{\prime}(\theta)+(1-t) q_{2}^{\prime}(\theta)
\end{array}\right), \\
|\operatorname{det}(J U(t, \theta))|=\mid\left(p_{1}(\theta)-q_{1}(\theta)\right)\left(t p_{2}^{\prime}(\theta)+(1-t) q_{2}^{\prime}(\theta)\right) \\
-\left(t p_{1}^{\prime}(\theta)+(1-t) q_{1}^{\prime}(\theta)\right)\left(p_{2}(\theta)-q_{2}(\theta)\right) \mid \\
=|t u(\theta)+v(\theta)| \equiv \pm(t u(\theta)+v(\theta)) \in \mathbb{P}_{1}([0,1]) \bigotimes \mathbb{T}_{2}([\alpha, \beta]),
\end{gathered}
$$

since

$$
\mathbb{T}_{1}([\alpha, \beta]) \ni u=\left(p_{1}-q_{1}\right)\left(p_{2}^{\prime}-q_{2}^{\prime}\right)-\left(p_{1}^{\prime}-q_{1}^{\prime}\right)\left(p_{2}-q_{2}\right),
$$




$$
\mathbb{T}_{2}([\alpha, \beta]) \ni v=\left(p_{1}-q_{1}\right) q_{2}^{\prime}+\left(q_{2}-p_{2}\right) q_{1}^{\prime},
$$

and the Jacobian determinant doesn't change sign

$$
|\operatorname{det}(J U(t, \theta))| \equiv \pm \operatorname{det}(J U(t, \theta)) .
$$

In fact, after a little algebra we obtain

$$
u(\theta)=u_{0}+u_{1} \cos (\theta)+u_{2} \sin (\theta)
$$

with

$$
\begin{aligned}
& u_{0}=\left(a_{11}-a_{21}\right)\left(b_{12}-b_{22}\right)+\left(a_{12}-a_{22}\right)\left(b_{21}-b_{11}\right), \\
& u_{1}=\left(b_{12}-b_{22}\right)\left(c_{11}-c_{21}\right)+\left(b_{21}-b_{11}\right)\left(c_{12}-c_{22}\right), \\
& u_{2}=\left(a_{11}-a_{21}\right)\left(c_{12}-c_{22}\right)+\left(a_{12}-a_{22}\right)\left(c_{21}-c_{11}\right),
\end{aligned}
$$

and

$$
v(\theta)=v_{0}+v_{1} \cos (\theta)+v_{2} \sin (\theta)+v_{3} \cos (\theta) \sin (\theta)+v_{4} \sin ^{2}(\theta)
$$

with

$$
\begin{aligned}
& v_{0}=b_{21}\left(a_{22}-a_{12}\right)+b_{22}\left(a_{11}-a_{21}\right), \\
& v_{1}=b_{21}\left(c_{22}-c_{12}\right)+b_{22}\left(c_{11}-c_{21}\right), \\
& v_{2}=a_{21}\left(c_{12}-c_{22}\right)+a_{22}\left(c_{21}-c_{11}\right), \\
& v_{3}=a_{12} a_{21}-a_{11} a_{22}+b_{11} b_{22}-b_{12} b_{21}, \\
& v_{4}=a_{12} b_{21}-a_{11} b_{22}+a_{21} b_{12}-a_{22} b_{11} .
\end{aligned}
$$

Remark 1 It is worth noticing that there is a simple geometric characterization of injectivity of the transformation $U$ in the interior of the rectangle, i.e., that the $\operatorname{arcs} P$ and $Q$ intersect each other only possibly at their endpoints, and any two segments $\left[P\left(\theta_{1}\right), Q\left(\theta_{1}\right)\right]$ and $\left[P\left(\theta_{2}\right), Q\left(\theta_{2}\right)\right]$ intersect each other only possibly at one of their endpoints.

In the case of a generalized sector corresponding to an arc of ellipse (or circle), we have that $P(\theta) \equiv C_{1}$, and the characterization above means that the vertex $C_{1}$ belongs to an admissible portion of the double-angle planar region between the two tangent lines at $Q(\alpha)$ and $Q(\beta)$, which contains the $\operatorname{arc} Q$; if the lines are parallel, this region is a strip.

We have two cases: $i$ ) the segment joining $C_{2}$ and the intersection point of the tangent lines, say $V$, does not intersect the arc: then, the admissible region is bounded, and coincides with the portion of the double-angle which has the arc in the boundary and contains $\left.C_{2} ; i i\right)$ the segment joining $C_{2}$ and $V$ intersects the arc: then, the admissible region is the (now unbounded) portion above plus the opposite part of the double-angle, that is an unbounded sector with vertex $V$. Observe that taking $C_{1}$ out of the admissible region, there are segments $\left[C_{1}, Q(\theta)\right]$ which intersect the $\operatorname{arc} Q$ in two distinct points; see Figure 1. 

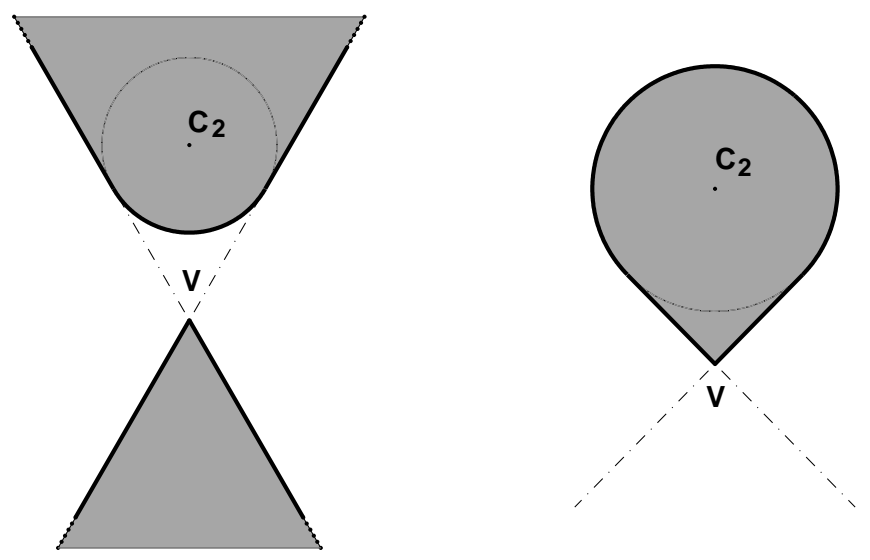

Figure 1: Admissible regions for the vertices of generalized sectors with respect to a circle arc; left: unbounded case; right: bounded case.

We can now state and prove the following

Proposition 2 Consider the planar domain generated by linear blending of two parametric arcs as in (5)-(7)

$$
\Omega=\{(x, y)=U(t, \theta),(t, \theta) \in[0,1] \times[\alpha, \beta], 0<\beta-\alpha \leq 2 \pi\},
$$

where the transformation $U(t, \theta)$ is injective for $(t, \theta) \in(0,1) \times(\alpha, \beta)$; cf. Remark 1. Then the following product Gaussian formula with $n^{2} / 2+\mathcal{O}(n)$ nodes holds

$$
\iint_{\Omega} f(x, y) d x d y=I_{n}(f)=\sum_{j=1}^{n+k+1} \sum_{i=1}^{\left\lceil\frac{n+h+1}{2}\right\rceil} W_{i j} f\left(x_{i j}, y_{i j}\right), \quad \forall f \in \mathbb{P}_{n}^{2},
$$

where $\mathbb{P}_{n}^{2}$ denotes the space of bivariate polynomials of total degree not greater than $n$, with $h=0$ if $u_{i}=0, i=0,1,2$, and $h=1$ otherwise, while $k=0$ if $u_{1}=u_{2}=0$ and $v_{i}=0, i=1, \ldots, 4, k=1$ if $v_{3}=v_{4}=0$ and at least one among $u_{1}, u_{2}, v_{1}, v_{2}$ is nonzero, and $k=2$ if $v_{3} \neq 0$ or $v_{4} \neq 0$; cf. (8)-(10).

In (12) we have

$$
\left(x_{i j}, y_{i j}\right)=U\left(t_{i}^{G L}, \theta_{j}+\mu\right), 0<W_{i j}=\left|\operatorname{det}\left(J U\left(t_{i}^{G L}, \theta_{j}+\mu\right)\right)\right| w_{i}^{G L} \lambda_{j},
$$


$\left\{\left(\theta_{j}+\mu, \lambda_{j}\right)\right\}$ being the angular nodes and weights of the trigonometric Gaussian formula (4) of degree of exactness $n+k$ on $[\alpha, \beta]$, and $\left\{\left(t_{i}^{G L}, w_{i}^{G L}\right)\right\}$ the nodes and weights of the Gauss-Legendre formula of degree of exactness $n+h$ on $[0,1]$.

Proof. First, observe that by (8)-(10) we have that $|\operatorname{det}(J U)|$ is a mixed algebraic-trigonometric polynomial belonging to the tensor-product space $\mathbb{P}_{h}([0,1]) \otimes \mathbb{T}_{k}([\alpha, \beta])$, where $h=0$ if $u_{i}=0, i=0,1,2$, and $h=1$ otherwise, while $k=0$ if $u_{1}=u_{2}=0$ and $v_{i}=0, i=1, \ldots, 4, k=1$ if $v_{3}=v_{4}=0$ and at least one among $u_{1}, u_{2}, v_{1}, v_{2}$ is nonzero, and $k=2$ if $v_{3} \neq 0$ or $v_{4} \neq 0$.

By injectivity of the transformation (7) we have that

$$
\iint_{\Omega} f(x, y) d x d y=\int_{0}^{1} \int_{\alpha}^{\beta} f(U(t, \theta))|\operatorname{det}(J U(t, \theta))| d \theta d t,
$$

where for $f \in \mathbb{P}_{n}^{2}$ the integrand in the right-hand side becomes a mixed algebraic-trigonometric polynomial, which belongs to the tensor-product space $\mathbb{P}_{n+h}([0,1]) \otimes \mathbb{T}_{n+k}([\alpha, \beta])$. Hence, we get exactness using the corresponding trigonometric Gaussian formula (4) in the variable $\theta$ and the classical Gauss-Legendre formula in the variable $t$. The overall number of nodes (and associated weights) is approximately $(n+h+1)(n+k+1) / 2=$ $n^{2} / 2+\mathcal{O}(n)$.

Remark 2 (Convergence rate). Concerning the convergence rate of the algebraic cubature formula (12)-(13), due to the positivity of the weights it is simple to show by standard arguments of quadrature theory that, for any fixed $k>0$ and sufficiently regular integrand $f$, we have the error estimate

$$
\iint_{\Omega} f(x, y) d x d y=I_{n}(f)+\mathcal{O}\left(n^{-k}\right) .
$$

Indeed, compacts of the form $\Omega=U([0,1] \times[\alpha, \beta])$, cf. (7), are all Jackson compacts, cf. [23].

We recall that the closure of an open bounded set, say $\Omega=\overline{\Omega^{\circ}} \subset \mathbb{R}^{d}$, is termed a Jackson compact if it admits a Jackson inequality, namely for each $k \in \mathbb{N}$ there exist a positive integer $m_{k}$ and a positive constant $c_{k}$ such that

$$
n^{k} \operatorname{dist}_{\Omega}\left(f, \mathbb{P}_{n}^{d}\right) \leq c_{k} \sum_{|i| \leq m_{k}}\left\|D^{i} f\right\|_{\Omega}, n>k, \quad \forall f \in C^{m_{k}}(\Omega)
$$

where $\operatorname{dist}_{\Omega}\left(f, \mathbb{P}_{n}^{d}\right)=\inf \left\{\|f-p\|_{\Omega}, p \in \mathbb{P}_{n}^{d}\right\}$. Examples of Jackson compacts are $d$-dimensional cubes (with $m_{k}=k+1$ ) and Euclidean balls (with $\left.m_{k}=k\right)$. 

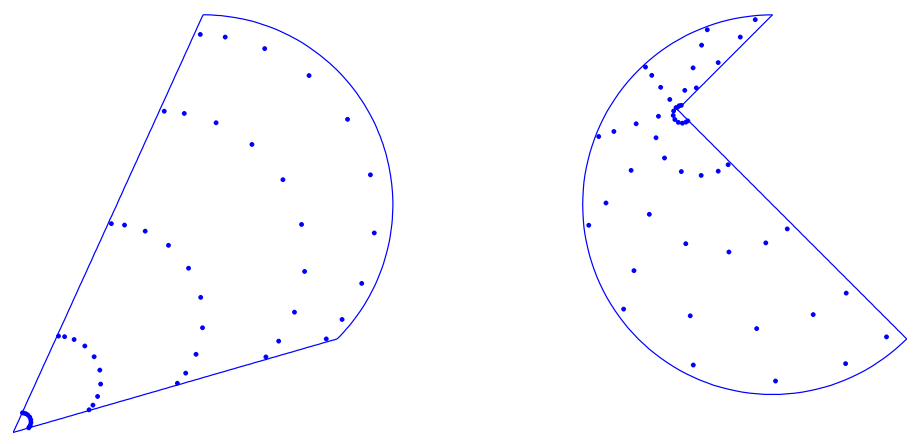

Figure 2: Cubature nodes for degree $n=8$ on two generalized sectors.

In [23], it is proved that if $\Omega$ is Whitney-regular and admits a Markov polynomial inequality, then it is a Jackson compact. Now, two deep results based on subanalytic geometry and pluripotential theory yield a conclusion. In fact, $\Omega=U([0,1] \times[\alpha, \beta])$ is the analytic image of a rectangle, thus it is a subanalytic compact subset of $\mathbb{R}^{2}$ and then it admits a Markov polynomial inequality; see [19] and the survey [22] for the relevant definitions and proofs. Moreover, $\Omega$ being subanalytic is also Whitney-regular (cf. [26]), and thus it is a Jackson compact.

Remark 3 (Standard circular/elliptical sections). It is worth observing that by formula (12) we can recover formulas recently obtained for standard sections of the disk, such as circular (annular) sectors, circular segments and zones, symmetric lenses (cf. [5]), with new possible representations arising. Several cubature formulas with polynomial exactness were previously known for the whole disk (cf., e.g., [3]) and for special disk sections such as complete annuli [21], but they were apparently missing in general cases.

For example, a circular segment (one of the two portions of a disk cut by a chord) can be represented at least in three different ways as linear blending of elliptical arcs. With no loss of generality consider a disk of radius $R$ centered at the origin, and the circular segment corresponding to 

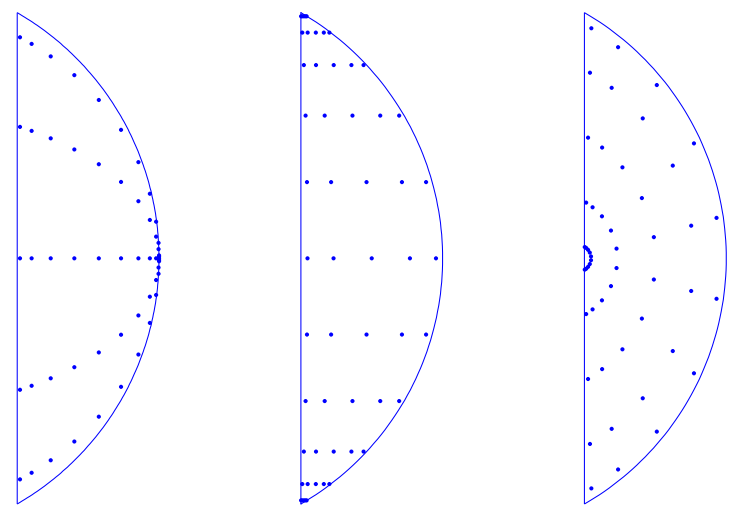

Figure 3: Cubature nodes for degree $n=8$ on a circular segment, corresponding to three different blendings.

an angular interval $[-\beta, \beta], 0<\beta<\pi$, where the midpoint of the chord is $(R \cos (\beta), 0)$.

With the first representation, $P(\theta)$ and $Q(\theta)$ are two symmetric halfarcs, $P(\theta)=(R \cos (\theta), R \sin (\theta)), Q(\theta)=(R \cos (\theta),-R \sin (\theta)), \theta \in[0, \beta]$. We have that $A_{1}=(R, 0), B_{1}=(0, R)$ and $C_{1}=(0,0)$, while $A_{2}=(R, 0)$, $B_{2}=(0,-R)$ and $C_{2}=(0,0)$. The resulting Jacobian determinant does not depend on $t$ since $u_{0}=u_{1}=u_{2}=0$, moreover $v_{4}=2 R^{2} \neq 0$; cf. (7)(10) (this generates the same cubature formula as the transformation used in [5]). Then $h=0$ and $k=2$ in (12) and the overall number of nodes is approximately $(n+1)(n+3) / 2$; here the nodes are disposed along vertical segments, see Figure 3-left.

With the second representation, valid for $0<\beta \leq \pi / 2, P(\theta)$ and $Q(\theta)$ are the chord and the whole arc, $P(\theta)=(R \cos (\beta), R \sin (\theta)), Q(\theta)=$ $(R \cos (\theta), R \sin (\theta)), \theta \in[-\beta, \beta]$. Then, $A_{1}=(0,0), B_{1}=(0, R)$ and $C_{1}=$ $(R \cos (\beta), 0)$, while $A_{2}=(R, 0), B_{2}=(0, R)$ and $C_{2}=(0,0)$. Again, the resulting Jacobian determinant does not depend on $t$ since $u_{0}=u_{1}=u_{2}=0$. Moreover $v_{4}=R^{2} \neq 0$, hence $h=0$ and $k=2$ in (12) and the overall number of nodes is approximately $(n+1)(n+3) / 2$; with this representation, the nodes are disposed along horizontal segments, see Figure 3-center.

But we have also a third representation as a generalized elliptical sector, with vertex at the midpoint (or any other point) of the chord. We have for example $P(\theta) \equiv(R \cos (\beta), 0)$ and $Q(\theta)=(R \cos (\theta), R \sin (\theta)), \theta \in[-\beta, \beta]$, that is $A_{1}=B_{1}=(0,0)$ and $C_{1}=(R \cos (\beta), 0)$, while $A_{2}=(R, 0), B_{2}=$ 
$(0, R)$ and $C_{2}=(0,0)$. Since $v_{3}=v_{4}=0$ but $u_{1}=-R^{2} \cos (\beta) \neq 0$, then $h=$ 1 and $k=1$ in (12) and the overall number of nodes is approximately $(n+$ $2)^{2} / 2$; the nodes are now disposed along rays stemming from the "vertex", see Figure 3-right.

Finally, in Figure 4, we show the distribution of cubature nodes on three standard sections of an ellipse: elliptical segment, (annular) sector and zone.

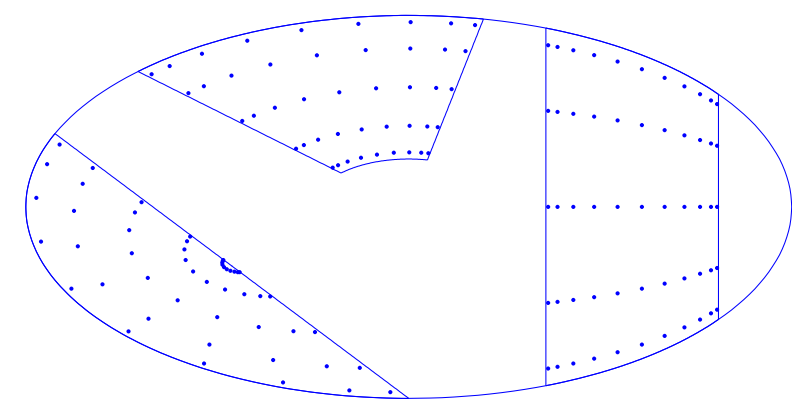

Figure 4: Cubature nodes for degree $n=8$ on three sections of an ellipse: elliptical segment, (annular) sector and zone.

\subsection{Implementation}

The efficient implementation of (12) rests on efficient methods for the computation of the underlying algebraic and trigonometric Gaussian formulas. A relevant difference between them is that when one has to compute integrals corresponding to different intervals in the same application, the GaussLegendre nodes and weights can be computed once (e.g. by Gautschi's OPQ suite [12]), whereas the trigonometric Gaussian angular nodes and weights have to be recomputed for every new angular subinterval. This can be done quite efficiently by the modified Chebyshev algorithm to compute the recurrence coefficients of the orthogonal polynomials with respect to the weight function $w(x)$ in (3), implemented by the Matlab function chebyshev.m in OPQ, followed by the classical spectral Golub-Welsch algorithm, implemented by gauss.m (an alternative method to compute the recurrence coefficients is the more general sub-range Jacobi polynomials approach, recently 
studied in [14]).

The modified Chebyshev algorithm requires that the moments (with respect to the same weight function) of another orthogonal polynomial basis are at hand, e.g., the Chebyshev moments

$$
m_{s}=\int_{-1}^{1} T_{s}(x) w(x) d x, \quad s=0,1, \ldots, 2 n+1 .
$$

For the even moments there is a known three-term nonhomogeneous linear recurrence [20] (the odd moments vanish), which is unstable forward but can be solved in a stable way by the associated $(n-1) \times(n-1)$ tridiagonal and diagonally dominant linear system, as soon as the first and last moment, say $m_{0}$ and $m_{2 n}$, are at hand. While the first moment $m_{0}$ is known, the last can be computed for example by Matlab's quadgk.m function for adaptive quadrature. We refer the reader to [5] for the details; the corresponding Matlab code is trigauss.m in [6].

In the present implementation we have improved the performance of trigauss.m. First, we have used the classical Olver algorithm [17] for the solution of the moment recurrence, which consists in solving the $(N-1) \times$ $(N-1)$ recurrence linear system with $m_{2 N}=0$ for a suitable $N>n$ (instead of computing directly $m_{2 n}$ ). This works well except for a neighborhood of $\omega=\pi$, where we retain the previous implementation. Moreover, we have pre-allocated some arrays in the OPQ function chebyshev.m, which reduces the computing time of the orthogonal polynomials recurrence coefficients.

Finally, we have adopted one of the fast variants of the Golub-Welsh algorithm, recently proposed in [16] for Gaussian rules with symmetric weight function, which give a remarkable speed-up with respect to the OPQ function gauss.m for degrees from the tens to the hundreds.

As a result, with the new version of trigauss.m we have obtained the speed-ups in Figure 5 for $n$ up to 200, with an agreement of the quadrature angular nodes and weights around $10^{-15}$. In practice, we are able to compute the cubature formula at degree $n=200$ in some milliseconds (Matlab 7.7.0 with an Athlon 64 X2 Dual Core $4400+2.40 \mathrm{GHz}$ processor). Such an improvement becomes particularly relevant when one has to compute the quadrature angular nodes and weights corresponding to a large number of different angular intervals, for example in the construction of discretization matrices for meshfree methods with compactly supported radial bases (cf., e.g., $[8,10,24])$.

In [7] we provide a Matlab function, named gqellblend.m, that computes nodes and weights of the algebraic cubature formula (12). This function automatically sets the parameters $h, k$ in (12) by checking the appropriate conditions on the coefficients $\left\{u_{i}\right\}$ and $\left\{v_{i}\right\}$ in (9)-(10). 


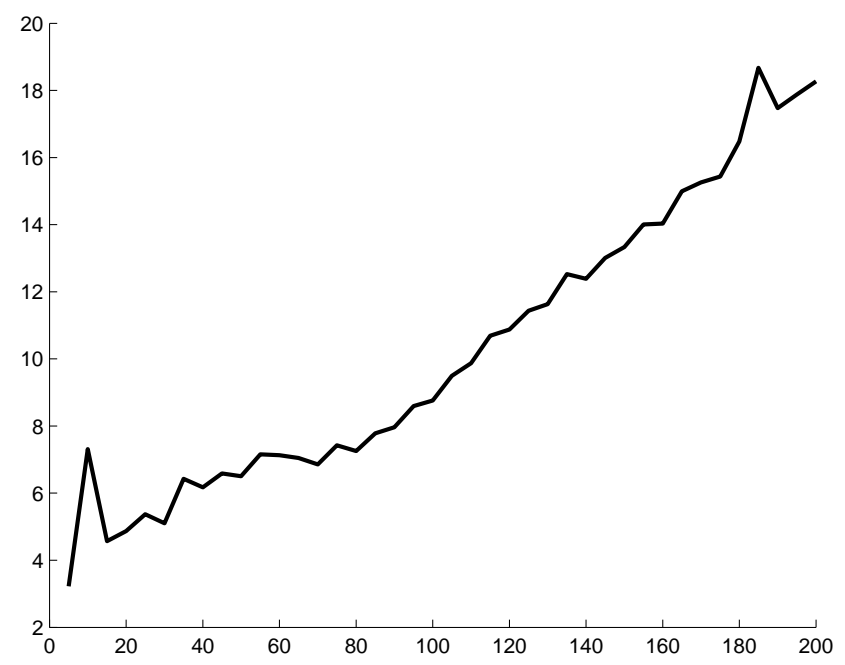

Figure 5: Average speed-up with the new version of trigauss.m in the computation of angular nodes and weights for trigonometric Gaussian quadrature in $[-\omega, \omega], \omega=\pi / 16, \pi / 8, \pi / 4, \pi / 2,3 \pi / 4,7 \pi / 8,15 \pi / 16$, for $n=5,10,15, \ldots, 200$.

\section{Applications}

We present now three of the several possible applications of our algebraic cubature formula by linear blending of elliptical arcs. The first two concern integration on nonstandard circular sections, with interesting connections to optical design and to meshfree projection methods. The last application concerns integration in the presence of singularities. All the numerical results have been obtained in Matlab 7.7.0 with an Athlon 64 X2 Dual Core 4400+ $2.40 \mathrm{GHz}$ processor.

\subsection{Application 1: sections of a lens}

As a first application we consider integration on a section of a circular lens (the intersection of two disks), cut by a circular arc or by a line.

The first case (cutting by a circular arc) corresponds to integration on the intersection of three disks. When the intersection is a (sub)lens, we can conveniently use the formula studied in [4]. Let us then restrict to the case when the intersection has three "vertices": it can be splitted, for example, into three generalized sectors, with the barycenter of the intersection as common vertex, generating cubature nodes with the structure appearing in Figure 6 . The possible case of an intersection with four "vertices" can be treated similarly. 


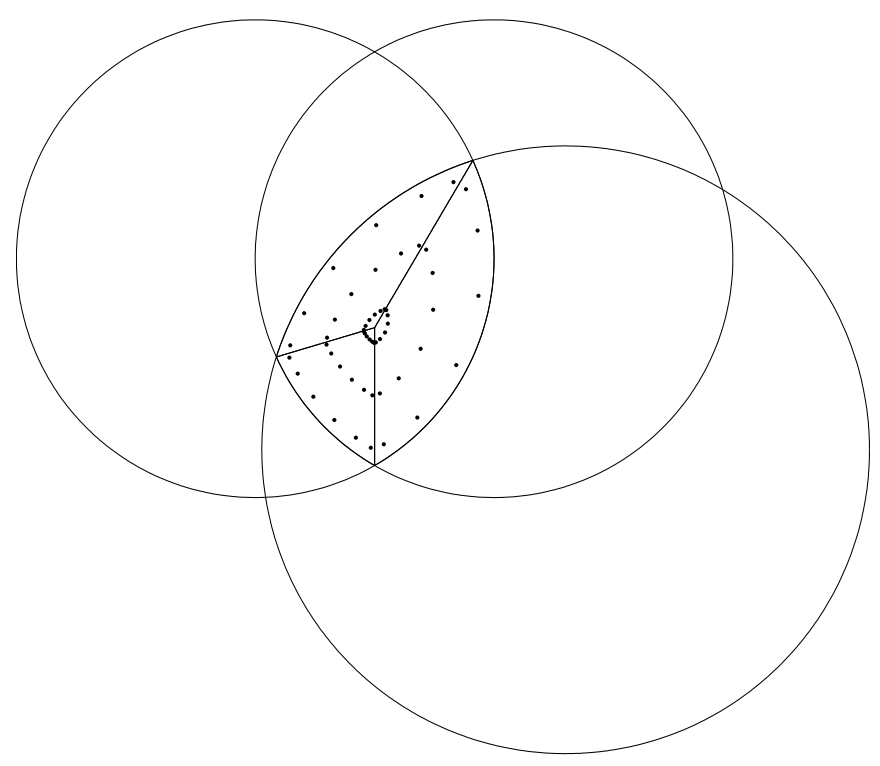

Figure 6: Cubature nodes for degree $n=4$ on the intersection of three disks.

The second case (cutting by a line) can arise, for example, in the framework of meshfree projection methods with radial compactly supported bases. Indeed, in the construction of Gram (least-squares) or stiffness (Galerkin) matrices, one has to integrate products of basis functions (or of their derivatives) on a large number of lenses, the supports intersections; cf., e.g., $[8,10,24]$ and references therein.

The integration problem becomes more difficult when interaction of a lens with the boundary of the approximation domains occurs. In the case of a piecewise linear boundary, a possible configuration is that of Figure 7. Observe that the integration domain can be conveniently splitted into two generalized sectors, with common vertex in the intersection of the lens axis with the line. A similar (but possibly more complicated) splitting approach can be used when the lens contains also vertices of the boundary polygonal line.

In Figure 8 we report, for the purpose of illustration, the relative errors in the numerical integration of the product of two Wendland's RBF (Radial Basis Functions),

$$
f(x, y)=\varphi\left(\left\|P-C_{1}\right\|_{2}\right) \varphi\left(\left\|P-C_{2}\right\|_{2}\right), \quad P=(x, y),
$$

on the lens section above, at a sequence of algebraic degrees of exactness, 


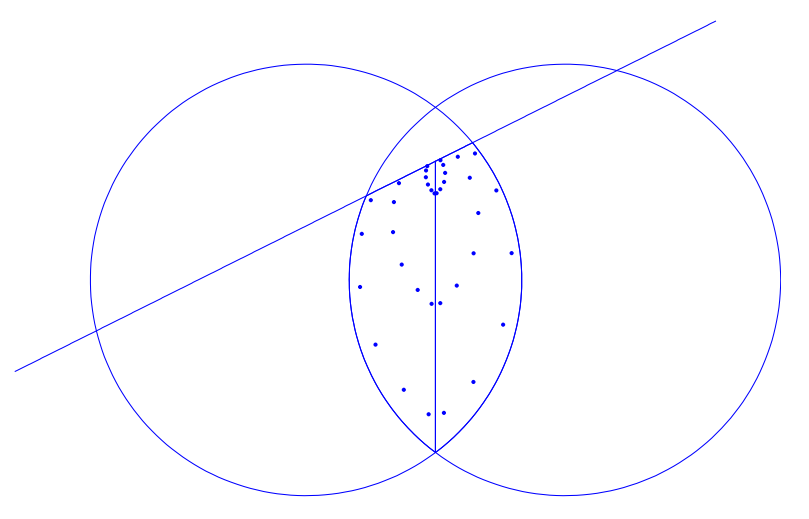

Figure 7: Cubature nodes for degree $n=4$ on a section of a lens.

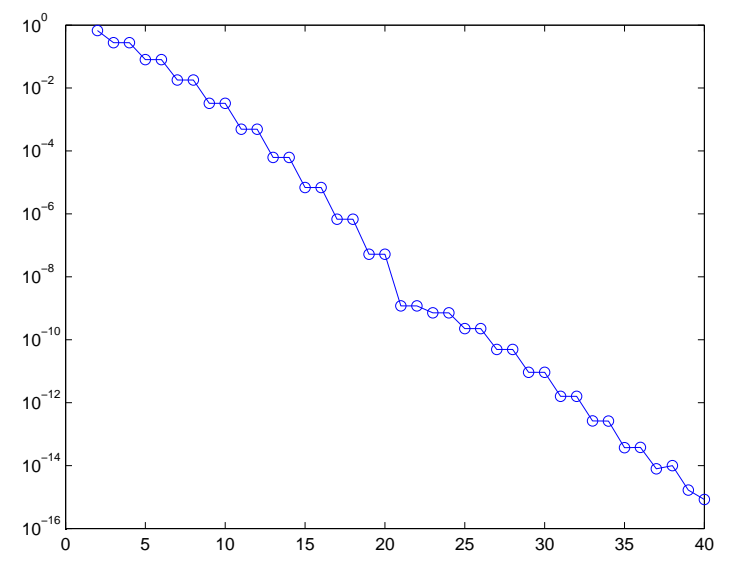

Figure 8: Relative errors in the numerical integration of the product of two Wendland RBF on the lens section, with respect to the degree.

where

$$
\varphi(r)=(1-r)_{+}^{4}(4 r+1),
$$

cf., e.g., $[10,28]$. The supporting disk centers are $C_{1}=(0,0)$ and $C_{2}=$ $(1.2,0)$, and the cutting line has equation $y=0.5 x+0.25$. Observe that even at the highest degrees (where we see an error close to machine precision) we have a computing time of the order of few milliseconds, whereas both, the standard Matlab's dblquad integrator and Shampine's TwoD integrator 
[25], need about 0.5 seconds to achieve an error of $10^{-6}$ and about 2 seconds for an error of $10^{-9}$ (both "suffer" at lower tolerances).

Observe that the integrand in (16) is $C^{\infty}$, since the centers of the supports (where the $\mathrm{RBF}$ are only $C^{2}$ ) are outside the integration domain. The case when the distance between the centers is smaller than the disk radius, and thus the centers enter the lens, will be treated in Application 3 of Section 3.1 .

\subsection{Application 2: obscured and vignetted pupils}

As a second example we consider special circular sections arising in the framework of optical design. The use of product Gaussian quadrature in optical design dates back to Forbes [11], who considered filled circular or elliptical apertures (pupils). More recently, Bauman and Xiao [1] have introduced quadrature methods based on prolate spheroidal wave functions [27], to treat situations where the pupil is obscured and vignetted (a feature that occurs, for example, in optical astronomy). We consider an example of circular pupil (the unit disk) which is obscured by a central smaller disk and clipped by a circular arc of larger radius, similar to that appearing in [1]; see Figure 9.

The resulting domain can be splitted into a standard annular sector (the unvignetted portion) and a vignetted region that cannot be treated directly as a linear blending in our present framework, since the arcs correspond to different angular intervals. Such a region, however, can be conveniently splitted for example into three generalized sectors, as shown in Figure 9. Collecting together nodes and weights of the corresponding algebraic cubature formulas, we obtain a composite cubature formula of polynomial degree of exactness $n$ on the obscured and vignetted pupil, with $4\left(n^{2} / 2+\mathcal{O}(n)\right)=2 n^{2}+\mathcal{O}(n)$ nodes and positive weights. We do not claim here that our composite cubature formula has a better performance with respect to that used in [1], but simply that, differently from other formulas, it is constructed to guarantee polynomial exactness at a given degree (this is possible because we have now at disposal subperiodic Gaussian trigonometric rules). In order to check its numerical exactness, in Table 1 we report the relative errors in the integration of the polynomial $(x+y)^{n}$ by the splitting just described, at a sequence of degrees.

\subsection{Application 3: managing singularities}

We present here a general purpose technique, to integrate in the presence of singularities, which can be relevant also in the framework of meshfree projection methods. 


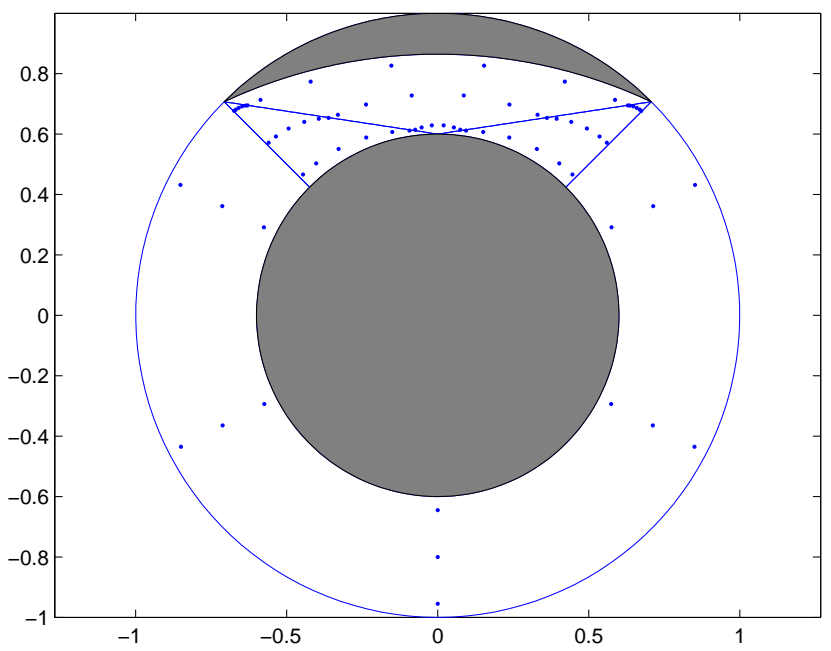

Figure 9: Cubature nodes for degree $n=4$ on an obscured and vignetted pupil, splitted into suitable generalized sectors.

Table 1: Relative errors in the integration of the polynomial $(x+y)^{n}$ on the obscured and vignetted pupil by the splitting of Figure 9 .

\begin{tabular}{cccccccc}
$n$ & 5 & 10 & 15 & 20 & 25 & 30 & 35 \\
\hline err & $4.8 \mathrm{e}-15$ & $1.5 \mathrm{e}-15$ & $4.1 \mathrm{e}-14$ & $1.6 \mathrm{e}-15$ & $1.7 \mathrm{e}-14$ & $8.2 \mathrm{e}-15$ & $4.1 \mathrm{e}-14$ \\
\hline
\end{tabular}

Consider the problem of integrating the function $f(x, y)=\left((x-a)^{2}+\right.$ $\left.(y-b)^{2}\right)^{k / 2}$ on the unit disk, with $k=1$ (Figure 11-top) or $k=-1$ (Figure 11-bottom), where $(a, b)$ is a point of the disk. Such a function is continuous for $k=1$, and unbounded but integrable for $k=-1$. If we apply a cubature formula that doesn't take into account the singularity, like e.g. that obtained by considering the disk as a zone, we see a very slow convergence for $k=1$, and essentially no convergence for $k=-1$. The behavior is similar with other representations of the disk and other formulas that "do not see" the singularity.

On the contrary, if we write the disk as a generalized sector centered at $(a, b)$, we get by construction a clustering of the nodes at the singularity (without "touching" it, since the cubature formula obtained by linear blending is open), and a satisfactory convergence rate even though the formula is not tailored to the specific function; see Figures 10-11. The error comparison is made with respect to the degree, since the formulas have roughly the same number of nodes at the same degree, $n^{2} / 2+\mathcal{O}(n)$. 

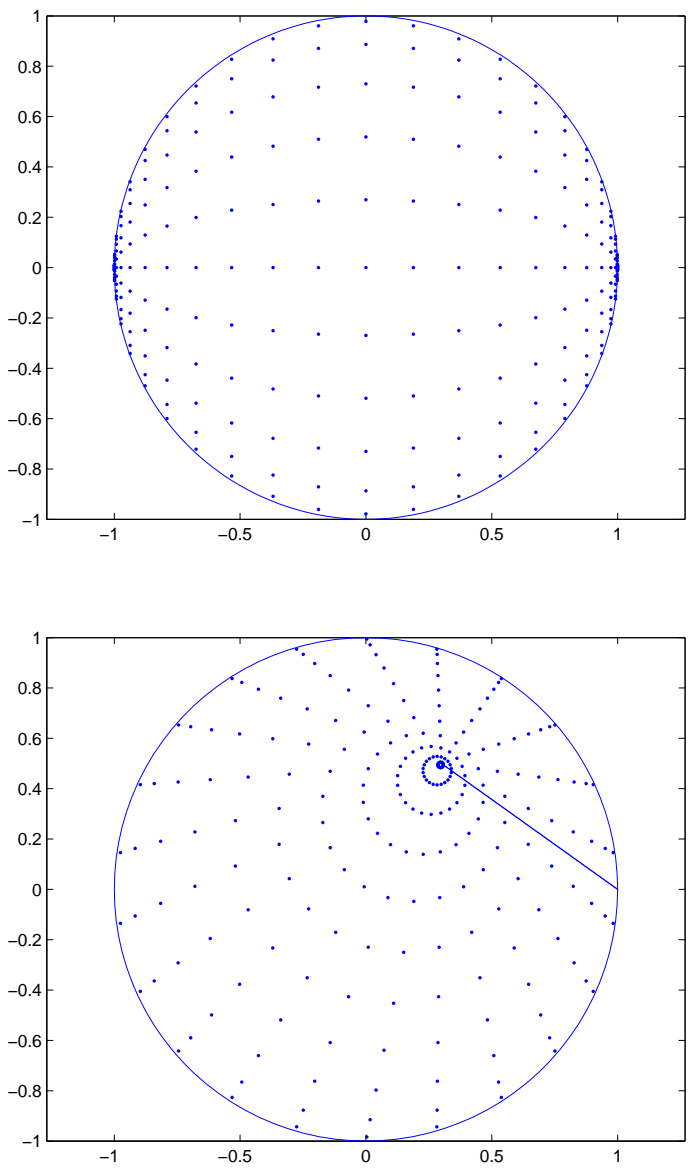

Figure 10: Distribution of the cubature nodes for degree $n=20$ on the disk, considered as a zone (top) or a generalized sector centered at $(a, b)$ (bottom).

It is even possible to treat a line of singularities by a suitable splitting. In Figure 12-top, we report the relative errors in the numerical integration of $f(x, y)=\sqrt{|x-y|}$, by our cubature formula on the unit disk considered as a zone or as the union of two circular segments (half-disks) with the common chord (diameter) $y=x$ (see Figure 12-bottom). This time the comparison is made with respect to the number of nodes, since for a fixed degree the second formula has roughly twice as many nodes. 

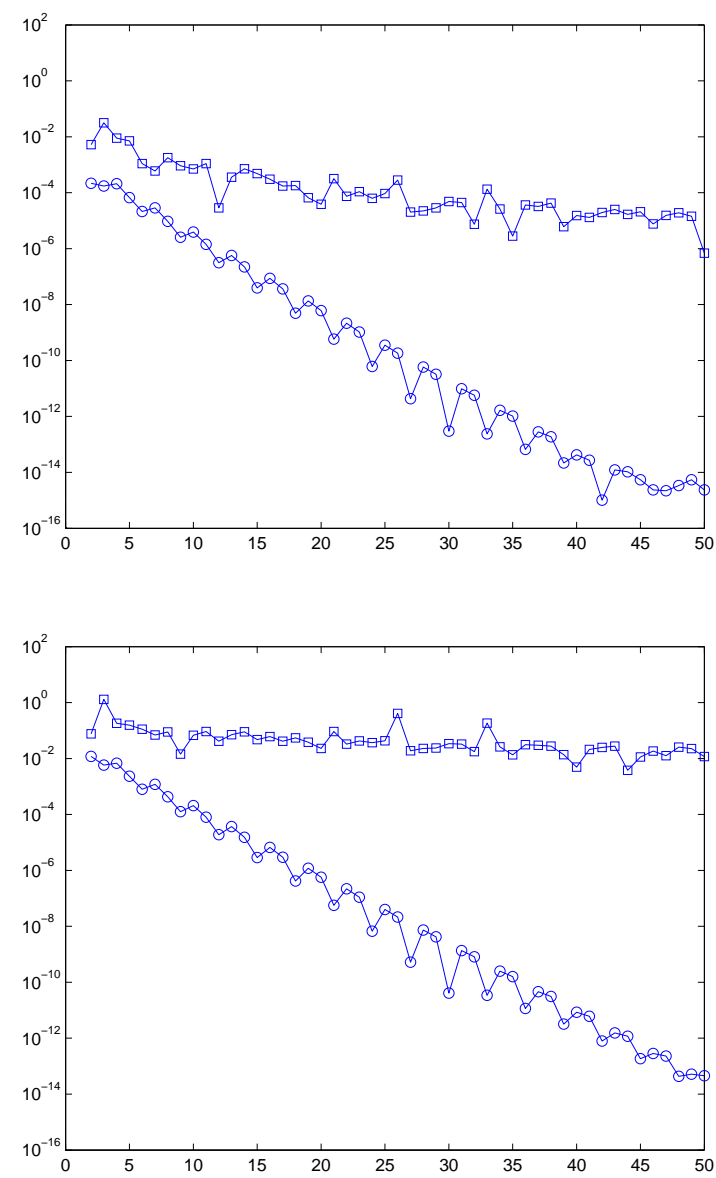

Figure 11: Relative errors with respect to the degree in the numerical integration of $f(x, y)=\left((x-a)^{2}+(y-b)^{2}\right)^{k / 2},(a, b)=(0.3,0.5), k=1$ (top), $k=-1$ (bottom), by our cubature formula on the unit disk considered as a zone (squares) or as a generalized sector centered at $(a, b)$ (circles).

As a last example we consider the problem of integrating the product of two compactly supported functions in the presence of singularities (of the derivatives), such as the product of Wendland RBF appearing in (16), with distance between the centers smaller than the radius.

In this case the centers, where the integrand is only $C^{2}$, enter the lens (intersection of the supports), and cubature formulas that "do not see" the singularity are not efficient. On the contrary, a suitable splitting into triangles and generalized sectors allows to construct a quite effective formula. We notice that the presence of some singularity at the center of the support is typical of basis functions used in meshfree methods.

In Figure 13-bottom we show the splitting of a half-lens (to which we 

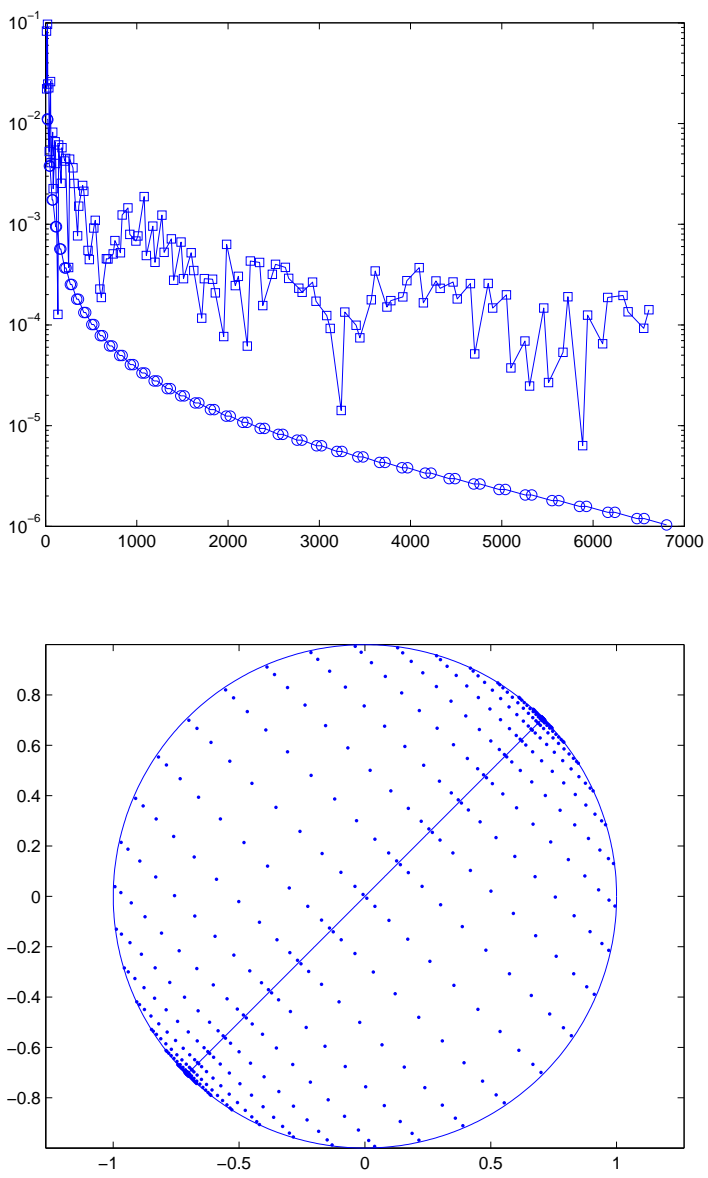

Figure 12: Top: Relative errors with respect to the number of nodes in the numerical integration of $f(x, y)=\sqrt{|x-y|}$, by our cubature formula on the unit disk considered as a zone (squares) or as the union of two circular segments with the common chord $y=x$ (circles); Bottom: Distribution of the cubature nodes for degree $n=20$ on the disk considered as the union of two circular segments.

can reduce the problem by symmetry), for $C_{1}=(0,0)$ and $C_{2}=(0.5,0)$, cf. (16). The relative errors in the numerical integration of the product of the Wendland RBF are reported in Figure 13-top, where we compare the formulas for the half-lens considered as a circular segment (cf. [4]), or splitted into two right triangles and a (concave) generalized sector, with a common vertex at the singular point, say $C_{2}$. We have chosen this splitting among other possible ones, for example a single triangle and the concave generalized sector with common vertex at $C_{2}$, since it has experimentally given the best results. 

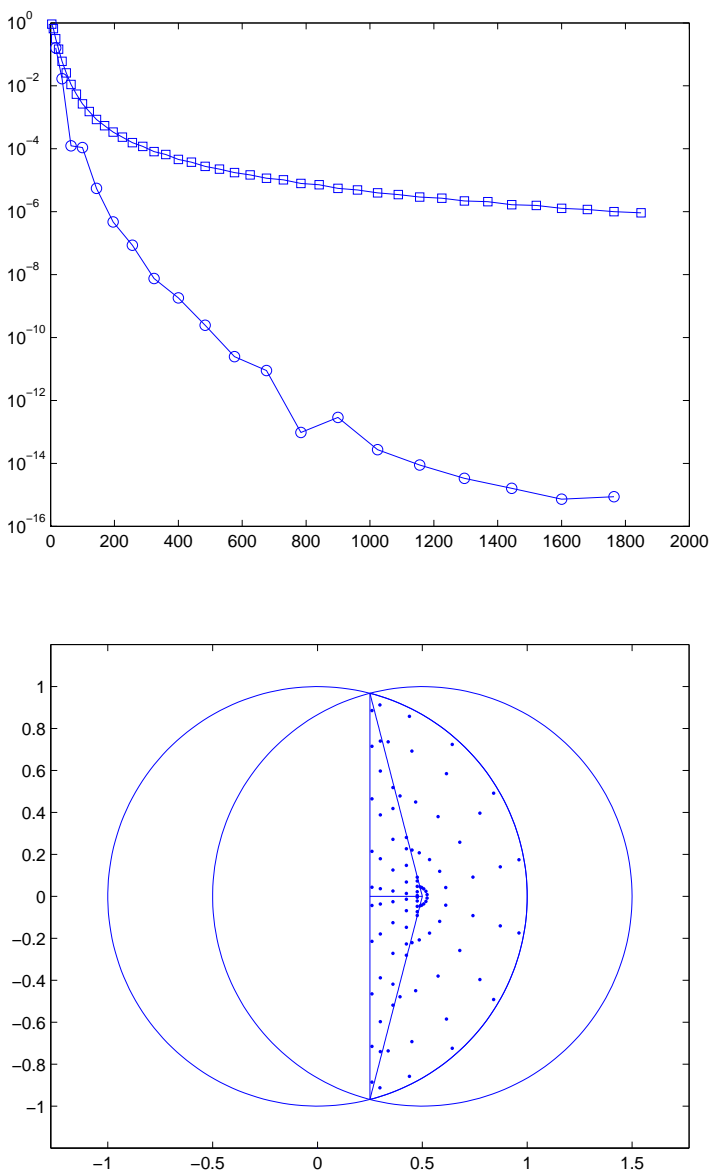

Figure 13: Top: Relative errors with respect to the number of nodes in the numerical integration of the product of two Wendland RBF on a halflens, considered as a circular segment (squares) or splitted as on the bottom (circles); Bottom: Distribution of the cubature nodes for degree $n=8$ on the splitted half-lens.

For the triangles we have used the classical Stroud's conical formula (cf. [15]) with node clustering at the common vertex, computed only once by symmetry. Though cubature formulas tailored to the specific singularity have been studied for triangles (cf., e.g., [15, §3.1]), we have used here a general clustering approach based on standard formulas, since we apply the same approach to the generalized sector. The overall number of nodes is $2\left(n^{2} / 4+\mathcal{O}(n)\right)+n^{2} / 2+\mathcal{O}(n)=n^{2}+\mathcal{O}(n)$.

Notice that the error of the splitting-based cubature formula becomes rapidly more accurate than the other one, with respect to the number of nodes, by several orders of magnitude. We stress that the splitting-based 
formula has a computing time of some milliseconds (small $n$ ) in the present degree range ( $n$ in the tens) and is able to reach machine precision, whereas the computing time of the standard Matlab integrators dblquad and TwoD is already in the seconds, to get an an error of about $10^{-9}$ (both "suffer" at lower tolerances).

A similar, though more complicated splitting approach, can be adopted also when the lens contains the centers and intersects the global approximation domain of the meshfree method (see Application 1 above).

\section{Conclusions}

Starting from a recent Gaussian-like quadrature formula with $n+1$ nodes (angles) and positive weights, exact for trigonometric polynomials of degree not greater than $n$ restricted to an angular interval $[-\omega, \omega]$ of length $2 \omega<2 \pi$ (subperiodic trigonometric quadrature), we have constructed a general cubature formula of product type, exact for polynomials of degree $n$ on planar domains corresponding to linear blending of two arcs of circle/ellipse. Such a formula generalizes several recent cubature formulas on standard circular sections, being applicable for example to sectors with vertex different from the disk center (see Figure 2). We provide the corresponding Matlab codes, where we have also optimized the underlying subperiodic trigonometric quadrature [7].

Among the numerous possible applications (we discuss three of them in Section 3), we quote and stress numerical integration of products of compactly supported functions with radial support, on piecewise linear sections of a planar circular lense (the intersection of the supports). Numerical examples related to compactly supported $\mathrm{RBF}$ are developed, for the purpose of illustration of the potential of the method (see Sections 3.1 and 3.3). Being able to take into account the interaction with a piecewise linear (or even a piecewise circular/elliptical) boundary, and to manage singularities by domain splitting and node clustering, we think that our arc-based blending approach could play a role in the implementation of Galerkin-like meshfree methods, concerning the still challenging aspect of efficiency in numerical integration.

\section{References}

[1] B. Bauman and H. Xiao, Gaussian quadrature for optical design with noncircular pupils and fields, and broad wavelength range, Proc. SPIE, 7652(2) (2010), 1-12.

[2] L. Bos and M. Vianello, Subperiodic trigonometric interpolation and quadrature, Appl. Math. Comput. 218 (2012), 10630-10638. 
[3] R. Cools and K.J. Kim, A survey of known and new cubature formulas for the unit disk, Korean J. Comput. Appl. Math. 7 (2000), 477-485.

[4] G. Da Fies and M. Vianello, Algebraic cubature on planar lenses and bubbles, Dolomites Res. Notes Approx. 5 (2012), 7-12.

[5] G. Da Fies and M. Vianello, Trigonometric Gaussian quadrature on subintervals of the period, Electron. Trans. Numer. Anal. 39 (2012), $102-112$.

[6] G. Da Fies and M. Vianello, Matlab functions for subperiodic trigonometric quadrature and for product Gaussian quadrature on circular sections, online at:

http://www.math.unipd.it/ marcov/CAAsoft.html.

[7] G. Da Fies, A. Sommariva and M. Vianello, Matlab functions for product Gaussian quadrature by linear blending of elliptical arcs, online at: http://www.math.unipd.it/ marcov/CAAsoft.html.

[8] S. De and K.-J. Bathe, The method of finite spheres with improved numerical integration, Comput. \& Structures 79 (2001), 218-231.

[9] G. Farin, Curves and surfaces for computer aided geometric design, 2nd ed., Academic Press, Boston, 1990.

[10] G. Fasshauer, Meshfree Approximation Methods with MATLAB, World Scientific, Singapore, 2007.

[11] G.W. Forbes, Optical system assessment of design: numerical ray tracing into the Gaussian pupil, JOSA A 5 (1988), 1943-1956.

[12] W. Gautschi, Orthogonal Polynomials: Computation and Approximation, Oxford University Press, New York, 2004.

[13] W. Gautschi, Orthogonal polynomials (in Matlab), J. Comput. Appl. Math. 178 (2005), 215-234, software online at:

http://www.cs.purdue.edu/archives/2002/wxg/codes.

[14] W. Gautschi, Sub-range Jacobi polynomials, Numer. Algorithms 61 (2012), 649-657.

[15] J.N. Lyness and R. Cools, A survey of numerical cubature over triangles, Proc. Sympos. Appl. Math., 48, Amer. Math. Soc., Providence, 1994.

[16] G. Meurant and A. Sommariva, Fast variants of the Golub and Welsch algorithm for symmetric weight functions, 2012, submitted. 
[17] F.W.J. Olver, Numerical solution of second order linear difference equations, J. Res. Nat. Bur. Standards 71B (1967), 111-129.

[18] E. Outerelo and J.M. Ruiz, Mapping degree theory, Graduate Studies in Mathematics, 108, American Mathematical Society, Providence, 2009.

[19] W. Pawłucki and W. Pleśniak, Markov's Inequality and $C^{\infty}$ Functions on Sets with Polynomial Cusps, Math. Ann. 275 (1986), 467-480.

[20] R. Piessens, Modified Clenshaw-Curtis integration and applications to numerical computation of integral transforms, Numerical integration (Halifax, N.S., 1986), 35-51, NATO Adv. Sci. Inst. Ser. C Math. Phys. Sci., 203, Reidel, Dordrecht, 1987.

[21] W.H. Pierce, Numerical integration over the planar annulus, J. Soc. Indust. Appl. Math. 5 (1957), 66-73.

[22] W. Pleśniak, Multivariate polynomial inequalities via pluripotential theory and subanalytic geometry methods, Banach Center Publ. 72 (2006), 251-261.

[23] W. Pleśniak, Multivariate Jackson Inequality, J. Comput. Appl. Math. 233 (2009), 815-820.

[24] D. Racz and T.Q. Bui, Novel adaptive meshfree integration techniques in meshless methods, Int. J. Numer. Meth. Engng. 90 (2012), 14141434 .

[25] L.F. Shampine, MATLAB program for quadrature in 2D, Appl. Math. Comput. 202 (2008), 266-274.

[26] J. Stasica, The Whitney condition for subanalytic sets, Zeszyty Nauk. Uniw. Jagiellon. Prace Mat. 23 (1982), 211-221.

[27] H. Xiao, V. Rokhlin and N. Yarvin, Prolate Spheroidal Wave Functions, Quadrature and Interpolation, Inverse Problems 17 (2001), 805-838.

[28] H. Wendland, Scattered data approximation, Cambridge University Press, Cambridge, 2005. 